\title{
An APOS Analysis of Students' Understanding of the Concept of a Limit of a Function
}

\author{
Aneshkumar Maharaj \\ School of Mathematical Sciences \\ University of KwaZulu-Natal \\ maharaja32@ukzn.ac.za
}

This article reports on a study which used the APOS (Action-Process-Object-Schema) theory framework to investigate university students' understanding of limits of functions. The relevant limit concepts were taught to undergraduate science students at a university in Kwazulu-Natal in South Africa. This paper reports on the analysis of students' responses to four types of questions on limits of functions. The findings of this study confirmed that the limit concept is one that students find difficult to understand, and suggests that this is possibly the result of many students not having appropriate mental structures at the process, object and schema levels.

In South Africa Grade 12 learners are exposed to an intuitive understanding of the limit of functions. This occurs in the context of the evaluation of limits of functions when finding derivatives from first principles of basic functions of the types $f(x)=b, f(x)=x, f(x)=x^{2}, f(x)=x^{3}$ and $f(x)=\frac{1}{x}$ (Department of Education, 2003). For this given context calculations for the limit of a function as $x$ approaches $a$, create the impression that this is the same as the value of the function at $a$. During the past eight years, this is what my interactions with first year university mathematics students indicated. When confronted with a problem to evaluate a limit of a function, many students simply proceed to find the corresponding function value; even when the limit or this function value does not exist. This indicated that there was a need to engage with a study on students' understanding of the concept of a limit of a function. The research questions for this study were:

- How should the teaching of the concept of a limit of a function be approached?

- What insights would an APOS analysis of students' understanding of the concept of a limit of a function reveal?

\section{Theoretical framework}

This study is based on APOS theory (Dubinsky \& McDonald, 2001). APOS theory proposes that an individual has to have appropriate mental structures to make sense of a given mathematical concept. The mental structures refer to the likely actions, processes, objects and schema required to learn the concept. Research based on this theory requires that for a given concept the likely mental structures need to be detected, and then suitable learning activities should be designed to support the construction of these mental structures.

Asiala, et al. (1996) proposed a specific framework for research and curriculum development in undergraduate mathematics education. The framework consists of the following three components: theoretical analysis, teaching, and observations and assessment of student learning. According to Asiala et al. (1996), APOS theory functions according to the paradigm illustrated in Figure 1. 
In this paradigm, theoretical analysis occurs relative to the researcher's knowledge of the concept in question and knowledge of APOS theory. This theoretical analysis helps to predict the mental structures that are required to learn the concept. For a given mathematical concept, the theoretical analysis informs the design and implementation of teaching. These are used for collection and analysis of data. The theoretical analysis guides the latter, which Figure 1 indicates could lead to a modification of the initial theoretical analysis of the given mathematical concept.

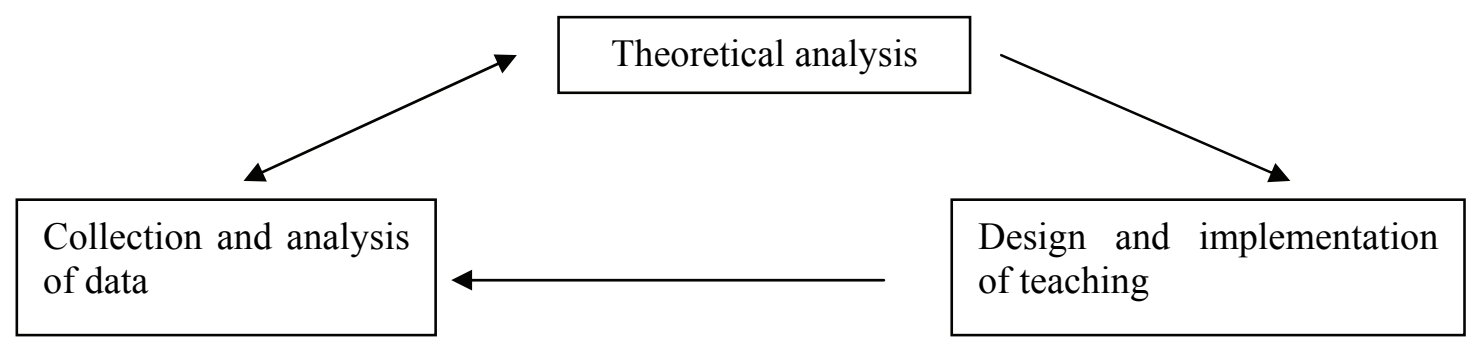

Figure 1: Paradigm: General research programme

\section{Description of the APOS/ACE teaching}

APOS Theory and its application to teaching practice are based on two general hypotheses developed to understand the ideas of Jean Piaget. In some studies (see, for example, Weller et al., 2003), these ideas were recast and applied to various topics in post-secondary mathematics. Piaget investigated the thinking of adolescents and adults, including research mathematicians. Those investigations led him to uncover common characteristics, specifically certain mental structures and mechanisms that guide concept acquisition (Piaget, 1970). APOS theory and its application to teaching practice are based on the following assumptions (Dubinsky, 2010):

- Assumption on mathematical knowledge: An individual's mathematical knowledge is his/her tendency to respond to perceived mathematical problem situations and their solutions by reflecting on them in a social context, and constructing or reconstructing mental structures to use in dealing with the situations.

- Hypothesis on learning: An individual does not learn mathematical concepts directly. He/she applies mental structures to make sense of a concept (Piaget, 1964). Learning is facilitated if the individual possesses mental structures appropriate for a given mathematical concept. If appropriate mental structures are not present, then learning the concept is almost impossible.

The above imply that the goal for teaching should consist of strategies for helping students build appropriate mental structures, and guiding them to apply these structures to construct their understanding of mathematical concepts. In APOS theory, the mental structures are actions, processes, objects, and schemas. In the following each of these are briefly described. Then the ACE teaching cycle; which constitutes the pedagogical strategies used to follow the hypothesis and the implication for teaching; is described.

After these general considerations, the assumption on mathematical knowledge is focused on by making an APOS analysis of the condition for the existence of a limit of a function. The result of this analysis is called a genetic decomposition. A genetic decomposition of a concept is a structured set of mental constructs which might describe how the concept can develop in the mind of an individual (Asiala, et. al., 1996). So, a genetic decomposition postulates the particular actions, processes, and objects that play a role in the construction of a mental schema for dealing with a given mathematical situation. The genetic decomposition arrived at for the limit of a function concept, is indicated in the methodology section.

\section{APOS theory}

The main mental mechanisms for building the mental structures of action, process, object, and schema are called interiorisation and encapsulation (Dubinsky, 2010; Weller et al., 2003). The mental structures of action, process, object, and schema constitute the acronym APOS. APOS theory postulates that a mathematical concept develops as one tries to transform existing physical or mental objects. The 
descriptions of action, process, object and schema; given below; are based on those given by Weller, Arnon and Dubinsky (2009).

- Action: A transformation is first conceived as an action, when it is a reaction to stimuli which an individual perceives as external. It requires specific teaching, and the need to perform each step of the transformation explicitly. For example, a student who requires an explicit expression to think about a limit of a function, $\lim _{x \rightarrow a} f(x)$, and can do little more than substitute values of $x$ close to $a$ for the variable in the expression $f(x)$ and manipulate it, is considered to have an action understanding of a limit of a function.

- Process: As an individual repeats and reflects on an action, it may be interiorised into a mental process. A process is a mental structure that performs the same operation as the action, but wholly in the mind of the individual. Specifically, the individual can imagine performing the transformation without having to execute each step explicitly. For example, an individual with a process understanding of the limit of a function; $\lim _{x \rightarrow a} f(x)$; will construct a mental process for values of $x$ close to $a$ and think in terms of inputs, possibly unspecified, and transformations of those inputs to produce outputs.

- Object: If one becomes aware of a process as a totality, realises that transformations can act on that totality and can actually construct such transformations (explicitly or in one's imagination), then we say the individual has encapsulated the process into a cognitive object. For example, for the limit of a function concept an individual may confront situations requiring him/her to apply various actions and/or processes. These could include thinking about an operation that takes two functions and produces a new function, such as in $\lim _{x \rightarrow 4^{+}} \frac{4-v}{|4-v|}$ or $\lim _{x \rightarrow 0^{-}}\left(\frac{1}{\sin x}\right)$. In order to operate on the one sided-limit of this new function, the process understanding must be encapsulated and converted to an object.

- Schema: A mathematical topic often involves many actions, processes, and objects that need to be organised and linked into a coherent framework, called a schema. It is coherent in that it provides an individual with a way of deciding, when presented with a particular mathematical situation, whether the schema applies. For example, the coherence might lie in the understanding that to determine the existence of a limit of a function, $\lim _{x \rightarrow a} f(x)$, the following must be considered: input values to the left and right of $a$, the corresponding output values, and a means of transforming elements of the inputs to elements of the outputs.

Explanations offered by an APOS analysis are limited to descriptions of the thinking which an individual might be capable. It is not asserted that such analyses describe what "really" happens in an individual's mind, since this is probably unknowable. Also, the fact that an individual possesses a certain mental structure does not mean that he or she will necessarily apply it in a given situation. This depends on other factors, for example managerial strategies, flexibility, prompts and emotional states. The main use of an APOS analysis is to point to possible pedagogical strategies. Data is collected to validate the analysis or to indicate that it must be reconsidered. For more details see Asiala et al. (1996), and Dubinsky and McDonald (2001).

\section{The ACE Teaching Cycle}

This pedagogical approach, based on APOS theory and the hypothesis on learning and teaching, is a repeated cycle consisting of three components: (A) activities, (C) classroom discussion, and (E) exercises done outside of class (Asiala, et. al., 1996). The activities, which form the first step of the cycle, are designed to foster the students' development of the mental structures called for by an APOS analysis. In the classroom the teacher guides the students to reflect on the activities and its relation to the mathematical concepts being studied. Students do this by performing mathematical tasks. They discuss their results and listen to explanations, by fellow students or the teacher, of the mathematical meanings of what they are working on. The homework exercises are fairly standard problems. They reinforce the knowledge obtained in the activities and classroom discussions. Students apply this knowledge to solve standard problems related to the topic being studied. The implementation of this approach and its effectiveness in helping students make mental constructions and learn mathematics has been reported in several research studies. A summary of early work can be found in Weller et al. (2003). 


\section{Literature review}

There are many studies on students' understanding of the concept of a limit of a function (e.g. Cornu, 1992; Davis \& Vinner, 1986; Li \& Tall, 1993; Maharajh, Brijlall, \& Govender, 2008; Monaghan, Sun, \& Tall, 1994; Tall, 1992; Tall \& Vinner, 1981; Williams, 1991). These studies indicate that students have difficulties with the concept of a limit of a function in the context of functions and continuity or series and sequences, and many of the difficulties encountered by students in dealing with other concepts; for example continuity, differentiability and integration; are related to their difficulties with limits.

Some researchers (Cornu, 1992; Sierpińska, 1987) reported that a high percentage of students have a static view of mathematics. Such students can only deal with a very specific calculation that is placed before them. Students with such a view will have difficulties with the limit of a function concept. The term procept is used to indicate that mathematical symbolism can ambiguously represent either a process or a concept (Monaghan et al., 1994). So, the symbol $\lim _{x \rightarrow a} f(x)$ is an example of a procept since it represents the process of getting to a specific value, or the value of the limit of the function itself. However, unlike the procepts of elementary mathematics, where an algorithm can be used to calculate the specific value of the concept, the limit value does not have a universal algorithm that works in all cases. Further, the limit of a function concept is not restricted to a finite computation that gives a definitive answer. This is precisely where the distinction between an action and a process comes in. It could be argued that once a calculation involves an infinite number of steps, it could only be understood through a process conception. A commonly cited difficulty that students have in constructing a process conception of limit of a function, is their perception of a limit of a function as something that is actually never attained (Cottrill, Nichols, Schwingendorf, Thomas, \& Vidakovic, 1996; Dubinsky, 2010).

It seems that many students perform poorly because they: (a) are unable to adequately handle information given in symbolic form which represent objects [abstract entities], for example functions, and (b) lack adequate schema or frameworks, which help to organise and link different objects (Maharaj, 2005). Giraldo, Carvalho and Tall (2003) distinguish between a description of a concept, which specifies some properties of that concept and the formal concept definition. Some descriptions commonly employed in the teaching of limits of functions include table of values, graphical and algebraic representations. Individually these involve limitations that do not fully reflect the mathematical situation. The teaching implication is that a variety of representations should be used, and to encourage students to engage with a flexibility of mathematical conceptions of $\lim _{x \rightarrow a} f(x)$.

\section{Participants and methodology}

\section{Participants and aim of module studied}

The participants for this study were 891 science students at a university in KwaZulu-Natal in 2010; about $66 \%$ of these were first year students. The students were studying a compulsory mathematics service module towards their Bachelor of Science degrees. Major subjects for these students varied over chemistry, physics, biology, zoology and pharmacy. The aim of the module studied is to introduce students to the fundamental principles, methods, procedures and techniques of mathematics and statistics as the language of science. These students attended their lectures in one of three timetable groups. I was the lecturer to all the students. This was the context for the theoretical analysis of the limit of a function concept.

\section{Theoretical analysis of the limit concept using an APOS approach, genetic decomposition}

The theoretical analysis indicated the type of mental structures of action, process, object, and schema relevant to both the limit of a function concept and types of limit problems that the participants encountered. Those mental structures were described in the section on APOS theory above. The following genetic decomposition was detected. At an action level a student confronted with the limit of a function, $\lim _{x \rightarrow a} f(x)$, can do little more than substitute values of $x$ close to $a$ for the variable in the expression $f(x)$ and manipulate it, and may or may not begin to see a pattern emerging. A process understanding of 
the limit of a function; $\lim _{x \rightarrow a} f(x)$; emerges as the student constructs a mental process for values of $x$ close to $a$ and thinks in terms of inputs, possibly unspecified, and transformations of those inputs to produce outputs. At the object level, the student sees this string as a totality and can perform mental or written actions on the one sided-limits of the given function, the process understanding is encapsulated and converted to an object, $\lim _{x \rightarrow a} f(x)$ which may or may not exist. At the schema level the actions, processes, and objects are organised and linked into a coherent framework. This framework includes possible techniques for evaluating $\lim _{x \rightarrow a} f(x)$; where $a$ could be $\infty$ or $-\infty$.

\section{The ACE teaching cycle}

This was informed by the theoretical analysis of the limit of a function concept and the types of limit problems that the students had to be exposed to. The key question for a 45 minute lecture session was: When does $\lim _{x \rightarrow a} f(x)$ exist? Activities were formulated and these were projected by use of a PC tablet. An example of an activity is given in Appendix A. A reasonable time was given for students to reflect and work on each activity; they were free to discuss with other students sitting beside them and to use the prescribed textbook. While students engaged with the activities I observed how they worked, their difficulties and aspects that required further explanations. These informed my explanations; using a PC tablet; to the class. Another 45 minute session was devoted to activities based on techniques for finding limits of functions, including limits at infinity. The activities and explanations incorporated use of graphical representations to answer questions on limits of functions, including limits of split-functions of the types given in symbolic notation; for types see questions 3 and 4 in Appendix A, and question 1 in Appendix B. Activities and classroom discussions were followed by homework exercises, which students had to work on as part of their tutorial requirements. A PC tablet was used to summarise the lecture-room discussions. These summaries were available to students, on the website for the module. During the 3 hour tutorial sessions about a week later, students were in groups of about 35 . In their groups they could further discuss the homework exercises with their tutors.

\section{Tools for collection of data}

About 3 weeks after the tutorials a multiple choice questions (MCQs) test was administered to 868 students. The questions set were similar to those for the activities and homework. Students were required to first work out the solutions in the space below each question and then to mark their choices on the multiple-choice-question cards. Each question was given a weighting of 3 marks. Students were informed that to discourage guessing, negative marking applied, i.e. -1 for each incorrect choice, which was a Science Faculty requirement for the use of MCQs at the university. The four questions on limits of functions are given in Appendix B. Note that the second question is based on the concept of continuity, which incorporates the concept of the existence of a limit of a function. The teaching for the section on continuity was similar to that outlined above, in that the ACE teaching cycle was followed. The options given for each of the MCQs were constructed bearing in mind the APOS levels of mental structures.

\section{Analysis, findings and discussion}

To represent the analysis, findings and discussion for each of the four questions in a reader friendly format, the following subheadings which describe the type of question are used:

- Limits of split-functions represented in symbolic form

- Continuity application of split-functions represented in symbolic form

- Limits of functions not defined at $x=a$

- Limits of rational functions at infinity

Under each of these subheadings the relevant test item and question analysis is given. The question analysis indicating number of student choices and the percentage (correct to one decimal place) for each of the four questions are indicated in Tables 1 to 4 below. In each of these tables "**" denotes the letter of the correct answer, for example D is the correct answer for Question 1. The Omit index gives the number of students who did not mark any of the alternatives, and the Bad index gives the number of students who marked more than one choice. 
Limits of split-functions represented in symbolic form

1. Let $f(x)= \begin{cases}x+2 & \text { if } x \leq 3 \\ 6-x & \text { if } x>3\end{cases}$

Then $\lim _{x \rightarrow 3} f(x)$
A) is 5
B) is 3
C) is 6
D) does not exist
E) is none of these

Table 1: Question 1 analysis of student choices $(N=868)$

\begin{tabular}{ccccccc}
\hline $\mathbf{A}$ & $\mathbf{B}$ & $\mathbf{C}$ & $\mathbf{D}^{*}$ & $\mathbf{E}$ & Omit index & Bad index \\
\hline 99 & 81 & 26 & 444 & 34 & 181 & 3 \\
$11,4 \%$ & $9,3 \%$ & $3 \%$ & $51,2 \%$ & $3,9 \%$ & $20,9 \%$ & \\
\hline
\end{tabular}

Question 1 is based on finding the limit of a split-function $f(x)$, as $x$ approaches a value in the domain where the function is split. The question analysis in Table 1, using the totals for choices A and B, indicates that 180 students conceptualised $\lim _{x \rightarrow 3} f(x)$ to be the same as one of the one-sided limits $\lim _{x \rightarrow 3^{+}} f(x)$ or $\lim _{x \rightarrow 3^{-}} f(x)$. In the APOS framework, using the genetic decomposition I arrived at, this means that those students' mental constructions were at best at the action level. This suggests that for evaluation of limits of split-functions, approximately $21 \%$ of the students had mental constructions developed up to the action level. The numbers for choice $\mathrm{C}$ suggests that a total of 26 students (approximately 3\%) had no idea of the basic technique for finding the limit of a split-function given in algebraic form; that is when the split-function is expressed in symbolic notation. A possible reason for 206 students selecting choices A, B or C is that they did not fully understand the concept of a splitfunction. This implies that it seems that approximately $23,7 \%$ of the students did not understand the concept of a split-function when such a function is represented in algebraic form. Table 1 also indicates that 444 students marked the correct choice for Question 1. In the context of my genetic decomposition this suggests that for evaluation of limits, of the type of split-functions under discussion, approximately $51,2 \%$ of the students had mental constructions developed up to the object level. Table 1 indicates that 34 students chose option E. This suggests approximately $4 \%$ of the students could have made calculation errors; and if this is accepted; their mental constructions were probably functioning at the object level. So it seems that approximately $55 \%$ of the students had appropriate mental structures in place for some sort of effective schema to evaluate the limit of a split-function $f(x)$ given in symbolic form, as $x$ approaches a value in the domain where the function is split. Table 1 also indicates that a large number of students (181, about 21\%) did not indicate any choice. One of the reasons for this is that they did not have any idea of how to work out the solution of such questions and did not guess, for fear of losing marks.

\section{Continuity application of split-functions represented in symbolic form}

2. Let $g(x)=\left\{\begin{array}{l}\frac{2 x^{2}-x-15}{x-3} \text { if } x \neq 3 \\ k x-1 \text { if } x=3\end{array}\right.$

The value of $k$ that will make the function $g$ continuous is
A) -3
B) 4
C) -15
D) 2
E) none of these 
Table 2: Question 2 analysis of student choices $(N=868)$

\begin{tabular}{ccccccc}
\hline $\mathbf{A}$ & $\mathbf{B} *$ & $\mathbf{C}$ & $\mathbf{D}$ & $\mathbf{E}$ & Omit index & Bad index \\
\hline 70 & 229 & 30 & 82 & 100 & 352 & 5 \\
$8,1 \%$ & $26,4 \%$ & $3,5 \%$ & $9,4 \%$ & $11,5 \%$ & $40,6 \%$ & \\
\hline
\end{tabular}

An analysis of Question 2 reveals that the point of discontinuity occurs at $x=3$. This is the crucial observation from the structure of the given split-function. The question is based on finding an unknown coefficient of one of the functions; in a split-function; which will make the entire function continuous on the interval $(-\infty, \infty)$. This requires continuity at $x=3$, which implies that the condition $\lim _{x \rightarrow 3} g(x)=g(3)$ must be satisfied. The type of mental conception required here involves the formulation of $\lim _{x \rightarrow 3} g(x)=g(3)$ as an equation which should be treated as an object. Further the successful use of this equation depends on an appropriately developed schema. This must incorporate conceptualisation of split-functions represented in symbolic form, as objects.

Note that in Question 2, if $x \neq 3$ then $g(x)=\frac{2 x^{2}-x-15}{x-3}$. So options A, C and D deal with distracters which were arrived at from this structure. If this is accepted then the question analysis in Table 2; using the totals for options A, C and D; indicates that 182 students (approximately $21 \%$ ) possibly had mental constructions which were not even at the action level. Table 2 also suggests that 229 students (approximately 26,4\%) possibly had appropriately developed schema for applications on continuity of split-functions represented in symbolic form. Since 100 students chose option E, this suggests approximately $11,5 \%$ of the students could have made calculation errors. If this is accepted then their mental constructions probably incorporated appropriate schema for applications on continuity of splitfunctions represented in symbolic form. So it seems that approximately $38 \%$ of the students had appropriate mental structures in place for some sort of effective schema for applications on continuity of split-functions represented in symbolic form. Table 2 also indicates that a large number of students 352 (approximately 40,6\%) did not indicate any choice. One of the reasons for this is that they did not have any idea of how to work out the solution of such questions and did not guess, for fear of losing marks.

\section{Limits of functions not defined at $x=a$}

3. The following limit is equal to:

$$
\lim _{x \rightarrow 36} \frac{\sqrt{x}-6}{x-36}
$$
A) $\frac{1}{6}$
B) 0
C) $-\infty$
D) $\frac{1}{12}$
E) $\infty$

Table 3: Question 3 analysis of student choices $(N=868)$

\begin{tabular}{ccccccc}
\hline $\mathbf{A}$ & $\mathbf{B}$ & $\mathbf{C}$ & $\mathbf{D}^{*}$ & $\mathbf{E}$ & Omit index & Bad index \\
\hline 68 & 269 & 46 & 254 & 103 & 124 & 4 \\
$7,8 \%$ & $31 \%$ & $5,3 \%$ & $29,3 \%$ & $11,9 \%$ & $14,3 \%$ & \\
\hline
\end{tabular}

Question 3 is based on the evaluation of limits of functions not defined at $x=a$. The limit of the function cannot be found by finding the corresponding function value. The technique here is to express the function in factorised form $\frac{\sqrt{x}-6}{(\sqrt{x}+6)(\sqrt{x}-6)}$, noting that $x \neq 36$ simplifies this to $\frac{1}{(\sqrt{x}+6)}$, and then finding $\lim _{x \rightarrow 36} \frac{1}{\sqrt{x}+6}$. Noting that $\frac{-6}{-36}=\frac{1}{6}$, Table 3 suggests that the 68 students (approximately 7,8\%) had mental constructions which were not even at an action level. The distracters given as options $\mathrm{B}, \mathrm{C}$ and $\mathrm{E}$ were designed for responses at a limited action level. If this is accepted then Table 3 suggests that 418 
students (approximately 48,2\%) possibly used mental constructions at some sort of action level. The 254 students (approximately 29,3\%) who marked the correct answer, conceptualised $\lim _{x \rightarrow 36} \frac{\sqrt{x}-6}{x-36}$ as an object. Further, using this object it seems they had appropriate schema to deal with the relevant imbedded and implied information. Table 3 suggests that 124 students (approximately 14,3\%) did not have any idea of how to work out the solution of such questions and did not guess, for fear of losing marks. So, it seems that at least $22 \%$ of the students had mental constructions not developed to any of the levels indicated in APOS Theory.

\section{Limits at infinity of rational functions}

4. The following infinite limit is equal to:

$$
\lim _{x \rightarrow \infty} \frac{-3 x^{2}+3 x-8}{-6 x^{2}+10}
$$
A) $-\frac{5}{4}$
B) 0
C) $\infty$
D) $\frac{1}{2}$
E) none of these

Table 4: Question 4 analysis of student choices $(N=868)$

\begin{tabular}{ccccccc}
\hline $\mathbf{A}$ & $\mathbf{B}$ & $\mathbf{C}$ & $\mathbf{D}^{*}$ & $\mathbf{E}$ & Omit index & Bad index \\
\hline 29 & 41 & 139 & 400 & 75 & 182 & 2 \\
$3,3 \%$ & $4,7 \%$ & $16 \%$ & $46,1 \%$ & $8,6 \%$ & $21 \%$ & \\
\hline
\end{tabular}

Question 4 is based on finding the limit at infinity of rational functions; the case where the polynomials in the numerator and denominator are of the same degree. Table 4 implies that the 180 students (approximately 20,7\%); those who chose options B and C; had no appropriate schema to deal with finding the limits at infinity of rational functions. Noting that $-\frac{5}{4}$ is the reciprocal of $\frac{-8}{10}$, Table 4 suggests that the 29 students (approximately 3,3\%) had mental constructions not even at the action level. Table 4 also implies that at least 400 students (approximately 46\%) had appropriately developed schema to deal with problems based on finding the limits at infinity of rational functions; in particular the case where the polynomials in the numerator and denominator are of the same degree. The 75 students (approximately $8,6 \%$ ) who chose option E, could have made calculation errors; and if this is accepted; their mental constructions probably incorporated appropriate schema for finding limits of rational functions, at infinity. So it seems that at most approximately $54,7 \%$ of the students had mental structures appropriately developed to some sort of effective schema for finding limits of rational functions, at infinity; in particular the case where the degree of the polynomial in the numerator is equal to that of the polynomial in the denominator.

The above analyses indicated that the types of MCQs used basically gave information on the highest potential mental structure levels of those students, according to APOS Theory. If this is accepted then the findings and analyses from Tables 1 to 4 are summarised in Table 5. Table 5 gives the highest potential levels of those students' mental structures for the four types of questions, indicated as subheadings in italics above, into which the four MCQs were classified.

For example, Table 5 suggests that for Question Type 1 (limits of split-functions represented in symbolic form) the responses of students indicated that their mental structures were $3 \%$ at not even an action level, $21 \%$ towards a potential process level, and $54,2 \%$ towards a potential schema level. Table 5 can be similarly interpreted for the other question types. The word potential is used since relevant mental structures at the action level should be in place for understanding to occur at the process level. According to APOS Theory, the challenge in teaching is to help such students to first attain the mental structures required for process understanding. 
Table 5: Percentage of responses towards highest potential levels according to mental structures

\begin{tabular}{cccc}
\hline Question Type & < Action level & Action $\rightarrow$ Process & Object $\rightarrow$ Schema \\
\hline $\mathbf{1}$ & 3 & 21 & 54,2 \\
$\mathbf{2}$ & 21 & & 37,9 \\
$\mathbf{3}$ & 7,8 & 48,2 & 29,3 \\
$\mathbf{4}$ & 3,3 & 2,7 & 54,7 \\
\hline
\end{tabular}

For Question 1 this would require the evaluation of both the one-sided-limits of the function, namely $\lim _{x \rightarrow 3^{+}} f(x)$ and $\lim _{x \rightarrow 3^{-}} f(x)$, then this needs to be encapsulated to form the object $\lim _{x \rightarrow 3} f(x)$. Once students have the relevant structures for object-level understanding, then the challenge is to help such students develop some sort of effective schema, which incorporates an organisational framework for using the actions, processes and objects to evaluate limits of a function $f(x)$ as $x$ approaches $a$. So teaching here would require suitable teaching on the following three types of limits of functions; using different representations for $f(x) ; \lim _{x \rightarrow 3^{+}} f(x), \lim _{x \rightarrow 3^{-}} f(x)$ and $\lim _{x \rightarrow 3} f(x)$. These representations should be verbal, graphical and algebraic. Since this study used algebraic and graphical representations, it seems that more emphasis should be on verbal representations. This could lead to graphical representations. For example, the following three activities could accomplish these:

\section{Activity 1}

A function $f$ behaves in the following way near $x=3$ :

As $x$ approaches 3 from the left, $f(x)$ approaches 2 .

As $x$ approaches 3 from the right, $f(x)$ approaches 1 .

For the above situation you are required to:

1. Draw a sketch to illustrate the behaviour of $f$ near $x=3$.

2. Write the $2^{\text {nd }}$ and $3^{\text {rd }}$ sentences in symbolic form.

3. Check that your symbolic form agrees with the sketch you drew.

4. Determine with reasons if $\lim _{x \rightarrow 3} f(x)$ exists.

\section{Activity 2}

Consider the split function $g(x)=\left\{\begin{array}{l}x+2 \text { if } x \leq 3 \\ 6-x \text { if } x>3\end{array}\right.$.

For this function you are required to:

1. Use the symbolic form to explain in your own words the behaviour of $g$ near $x=3$.

2. Use the symbolic form to draw the graph of $g$.

3. Evaluate $\lim _{x \rightarrow 3^{-}} g(x)$ and $\lim _{x \rightarrow 3^{+}} g(x)$.

4. Determine with reasons if $\lim _{x \rightarrow 3} g(x)$ exists.

\section{Activity 3}

1. Explain in your own words, for a function $h$, what is meant by " $\lim _{x \rightarrow a} h(x)$ exists".

2. Explain the procedure to find the limit of $\lim _{x \rightarrow a} h(x)$, where $h(x)$ is a split-function given in symbolic form.

Similarly for the other three question types, the interpretation of Table 5 can be used to formulate activities to help students develop the relevant mental structures. 


\section{Conclusions}

Useful insight into the relevant mental structures towards which teaching should focus was revealed by the APOS genetic decomposition of the limit of a function concept. The findings of this study confirmed that the limit of a function concept is one that students find difficult to understand, and suggests that this is possibly the result of many students not having appropriate mental structures at the process, object and schema levels. It seems that my genetic decomposition was adequate. However, my reflections on the teaching design indicated that more time needs to be devoted to helping students develop the mental structures at the process, object and schema levels. This implies that teaching should focus on (1) verbal and graphical approaches to finding limits of functions; including split-functions in symbolic form, (2) unpacking of structures given in symbolic form, and (3) modelling possible schema. A graphical approach should facilitate the development of mental structures at the process and object levels, while a focus on symbolic structures should aid object conceptions. If schemas organise and link the relevant actions, processes and objects then this should be a part of the teaching. The impact of such a focus on teaching will require further research.

\section{References}

Asiala, M., Brown, A., De Vries, D. J., Dubinsky, E., Mathews, D., \& Thomas, K. (1996). A framework for research and development in undergraduate mathematics education. Research in Collegiate Mathematics Education, 2, 1-32.

Cornu, B. (1992). Limits. In D. Tall (Ed.), Advanced mathematical thinking (pp. 153-166). Dordrecht: Kluwer Academic Publishers.

Cottrill, J., Nichols, D., Schwingendorf, K., Thomas, K., \& Vidakovic, D. (1996). Understanding the limit concept: Beginning with a coordinated process schema. Journal of Mathematical Behavior, 15(2), 167-192. Retrieved from http://homepages.ohiodominican.edu/ cottrilj/concept-limit.pdf

Davis, R. B., \& Vinner, S. (1986). The notion of limit: Some seemingly unavoidable misconception stages. Journal of Mathematical Behavior, 5, 281-303.

Department of Education. (2003). National curriculum statement Grades 10-12 (Schools): Mathematics. Pretoria: Department of Education.

Dubinsky, E. (2010, January). The APOS theory of learning mathematics: Pedagogical applications and results. Paper presented at the Eighteenth Annual Meeting of the Southern African Association for Research in Mathematics, Science and Technology Education. Durban, South Africa.

Dubinsky, E., \& McDonald, M. A. (2001). APOS: A constructivist theory of learning in undergraduate mathematics education research. In D. Holton (Ed.), The teaching and learning of mathematics at university level: An ICMI study (pp. 275-282). Dordrecht: Kluwer Academic Publishers. Retrieved from http://www.math.kent.edu/ edd/ICMIPaper.pdf

Giraldo, V., Carvalho, L. M., \& Tall, D. O. (2003). Descriptions and definitions in the teaching of elementary calculus. In N. A. Pateman, B.J. Dougherty, \& J. Zilliox (Eds.), Proceedings of the 27th Conference of the International Group for the Psychology of Mathematics Education (Vol. 2, pp. 445-452), Honolulu HI: Center for Research and Development Group, University of Hawaii. Retrieved from http://www.warwick.ac.uk/staff/David.Tall/pdfs/dot2003d-giraldo-carv-pme.pdf

Li, L., \& Tall, D. (1993). Constructing different concept images of sequences and limits by programming. In I. Hirabayashi, N. Nohda, K. Shigematsu, \& F. Lin (Eds.), Proceedings of the 17th Conference of the International Group for the Psychology of Mathematics Education (Vol 2, pp. 41-48). Tsukuba, Japan. Retrieved from http://www.warwick.ac.uk/staff/David.Tall/pdfs/dot1993e-lan-li-pme.pdf

Maharaj, A. (2005). Investigating the Senior Certificate Mathematics examination in South Africa: Implications for teaching. (Unpublished doctoral dissertation). University of South Africa, Johannesburg.

Maharajh, N., Brijlall,D., \& Govender, N. (2008). Preservice mathematics students' notions of the concept definition of continuity in calculus through collaborative instructional design worksheets. African Journal of Research in Mathematics, Science and Technology Education, 12, 93-108.

Monaghan, J., Sun, S., \& Tall, D. (1994). Construction of the limit concept with a computer algebra system. In J. P. Ponte \& J. F. Matos (Eds.), Proceedings of the 18th Conference of the International Group for the Psychology of Mathematics Education (Vol. 3, pp. 279-286). Lisbon: University of Lisbon. Retrieved from http://www.warwick.ac.uk/staff/David.Tall/pdfs/dot1994c-monhn-sun-pme.pdf

Piaget, J. (1964). Development and learning. Journal of Research in Science Teaching, 2, 176-180. 
Piaget, J. (1970). Piaget's theory (Translated by G. Cellerier and Jonas Langer; with the assistance of B. Inhelder and H. Sinclair). In P. H. Mussen (Ed.), Carmichael's manual of child psychology, Vol. 1 (3rd ed.) (pp. 703-732). New York: Wiley \& Sons.

Sierpińska, A. (1987). Humanities students and epistemological obstacles related to limits. Educational Studies in Mathematics, 18, 371-397.

Tall, D. (1992). The transition to advanced mathematical thinking: Functions, limits, infinity and proof. In D. A Grouws (Ed.), Handbook of research on mathematics teaching and learning (pp. 495-511). New York: Macmillan Publishing.

Tall, D., \& Vinner, S. (1981). Concept image and concept definition in mathematics with particular reference to limits and continuity. Educational Studies in Mathematics, 12, 151-169.

Weller, K., Clark, J., Dubinsky, E., Loch, S., McDonald, M., \& Merkovsky, R. (2003). Student performance and attitudes in courses based on APOS theory and the ACE teaching cycle. In A. Selden, E. Dubinsky, G. Harel, \& F. Hitt (Eds.), Research in collegiate mathematics education V (pp. 97-131). Providence, Rhode Island: American Mathematical Society.

Weller, K., Arnon, I., \& Dubinsky, E. (2009). Preservice teachers' understanding of the relation between a fraction or integer and its decimal expansion. Canadian Journal of Science, Mathematics and Technology Education, 9(1), 5-28.

Williams, S. R. (1991). Models of limit held by college calculus students. Journal for Research in Mathematics Education, 22, 219-236. 
Appendix A: An example of an activity

A. When does a limit exist?

1. Let $f(x)=\frac{x^{2}-1}{x-1}$. Does $\lim _{x \rightarrow 1} f(x)$ exist?

2. When does $\lim _{x \rightarrow a} f(x)$ exist?

3. Find the following limits

$3.1 \lim _{x \rightarrow 2} h(x)$ if $h(x)=\frac{x^{2}+4}{x-2}$

$3.2 \quad \lim _{x \rightarrow 0} \frac{|x|}{x}$

4. Refer to the graph gf $g(x)$.
4.1 Does $\lim _{x \rightarrow 3} g(x)$ exist?
42 Does $\lim _{x \rightarrow 2} g(x)$ exist?

Appendix B: The four multiple choice questions

1. Let $f(x)= \begin{cases}x+2 & \text { if } x \leq 3 \\ 6-x & \text { if } x>3\end{cases}$

Then $\lim _{x \rightarrow 3} f(x)$
A) is 5
B) is 3
C) is 6
D) does not exist
E) is none of these

2. Let $g(x)=\left\{\begin{array}{l}\frac{2 x^{2}-x-15}{x-3} \text { if } x \neq 3 \\ k x-1 \text { if } x=3\end{array}\right.$

The value of $k$ that will make the function $g$ continuous is
A) -3
B) 4
C) -15
D) 2
E) none of these

3. The following limit is equal to:

$\lim _{x \rightarrow 36} \frac{\sqrt{x}-6}{x-36}$
A) $\frac{1}{6}$
B) 0
C) $-\infty$
D) $\frac{1}{12}$
E) $\infty$

4. The following infinite limit is equal to:

$\lim _{x \rightarrow \infty} \frac{-3 x^{2}+3 x-8}{-6 x^{2}+10}$
A) $-\frac{5}{4}$
B) 0
C) $\infty$
D) $\frac{1}{2}$
E) none of these 